\title{
FATOU'S LEMMA IN INFINITE-DIMENSIONAL SPACES
}

\author{
NICHOLAS C. YANNELIS
}

(Communicated by R. Daniel Mauldin)

\begin{abstract}
Employing recent results of M. Ali Khan we provide an infinitedimensional version of the Fatou Lemma, which includes as a special case the result of Khan and Majumdar [15].
\end{abstract}

1. Introduction. Recently in an interesting paper, Khan and Majumdar [15] proved an approximate version of the well-known Fatou Lemma, for a separable Banach space. The purpose of this paper is to provide another approximate version of the Fatou Lemma which includes as a special case the result of Khan and Majumdar [15] (a more detailed comparison with their work is given in §5). Moreover, by adding an extra assumption to those of Khan and Majumdar, an exact version of Fatou's Lemma in infinite-dimensional spaces is also established.

The Fatou Lemma (see for instance Dunford and Schwartz [8, p. 152]), in addition to its significance in mathematics, has played an important role in mathematical economics. In particular, it was used by Aumann to prove the existence of a competitive equilibrium for an economy with an atomless measure space of traders and a finite-dimensional commodity space. Different versions of the Fatou Lemma in $n$-dimensions have been obtained by Aumann [2], Schmeidler [18], Hildenbrand and Mertens [10], and Artstein [1]. These results have found very interesting applications in economic theory (see, for example, Hildenbrand [9]).

Recently however, work in economies with a measure space of traders and infinitely many commodities necessitated an infinite-dimensional version of the Fatou Lemma (see for instance Yannelis [20, Remark 6.5]). More specifically, the desirable theorem was an infinite-dimensional version of Schmeidler's extension of the Fatou Lemma. Khan and Majumdar [15] tackled this interesting problem by employing some results of Khan [13]. They first obtained an approximate version of the Fatou Lemma for a separable Banach space. The approximate nature of their result arises from the fact that the Fatou Lemma is false in infinite dimensions. In particular, Uhl's [19] counterexample (see also [5, p. 262]) on the failure of the Lyapunov Theorem in infinite-dimensional spaces can be easily modified to indicate this as shown in Rustichini [17]. In this paper we build on Khan's recent work on integration in Banach spaces to provide an alternative approximate version of the Fatou Lemma. In particular, our Main Theorem is an approximate version of the Fatou Lemma for a separable Banach space or a Banach space whose dual has the Radon-Nikodým Property (RNP). Moreover, with additional assumptions we show

Received by the editors August 20, 1986 and, in revised form, February 2, 1987.

1980 Mathematics Subject Classification (1985 Revision). Primary 28A20, 46B22, 90A99.

Key words and phrases. Radon-Nikodým Property, Diestel's Theorem on weak compactness, Fatou's Lemma, approximate version of Lyapunov's Theorem, integral of a correspondence, integration preserves upper-semicontinuity, measurable selection. 
that an exact version of the Fatou Lemma can be obtained. Our Main Theorem enables us to prove easily that integration preserves upper-semicontinuity, i.e., if a correspondence taking values in a separable Banach space is upper-semicontinuous (u.s.c.) so is its integral.

The paper is organized as follows. $\S 2$ contains notation and definitions. The Main Theorem and its proof are given in $\S 3$. In $\S 4$ we show that integration preserves upper-semicontinuity. Finally, in $\S 5$ a comparison of our work with that of Khan and Majumdar [15] is given.

\section{Notation and definitions.}

2.1 Notation. $2^{A}$ denotes the set of all nonempty subsets of the set $A$.

$\overline{\operatorname{con}} A$ denotes the closed convex hull of $A$.

If $A \subset X$, where $X$ is a Banach space, $\operatorname{cl} A$ denotes the norm closure of $A$.

$\varnothing$ denotes the empty set.

If $Y$ is a linear topological space, its dual is the space $Y^{*}$ of all continuous linear functionals on $Y$, and if $p \in Y^{*}$ and $x \in Y$ the value of $p$ at $x$ is denoted by $\langle p, x\rangle$.

Finally if $Y$ is a Banach space and $F_{n}(n=1,2, \ldots)$ is a sequence of nonempty subsets of $Y$ we will denote by Ls $F_{n}$ the set of its weak limit superior points, i.e., Ls $F_{n} \equiv \mathrm{w}-\varlimsup_{n \rightarrow \infty} F_{n}=\left\{y \in Y: y=\mathrm{w}-\lim y_{k}, y_{k} \in F_{n_{k}}, k=1,2, \ldots\right\}$.

2.2 Definitions. Let $X$ and $Y$ be sets. The graph of the correspondence $\phi: X \rightarrow 2^{Y}$ is denoted by $G_{\phi}=\{(x, y) \in X \times Y: y \in \phi(x)\}$. Let $(T, \tau, \mu)$ be a complete finite measure space (i.e., $\mu$ is a real-valued, nonnegative countably additive measure defined on a complete $\sigma$-field $\tau$ of subsets of $T$ such that $\mu(T)<$ $\infty)$ and $Y$ be a Banach space. The correspondence $\phi: T \rightarrow 2^{Y}$ is said to have a measurable graph if $G_{\phi} \in \tau \otimes B(Y)$, where $B(Y)$ denotes the Borel $\sigma$-algebra on $Y$ and $\otimes$ denotes the product $\sigma$-field. The correspondence $\phi: T \rightarrow 2^{Y}$ is said to be lower measurable if for every open subset $V$ of $Y$ the set $\{t \in T: \phi(t) \cap V \neq \varnothing\}$ is an element of $\tau$. For the relationship between the above notions of measurability see $[11]$.

Following Diestel and Uhl [5] we define the notion of a Bochner integrable function. Let $(T, \tau, \mu)$ be a finite measure space and $Y$ be a Banach space. A function $f: T \rightarrow Y$ is called simple if there exist $y_{1}, y_{2}, \ldots, y_{n}$ in $Y$ and $\alpha_{1}, \alpha_{2}, \ldots, \alpha_{n}$ in $\tau$ such that $f=\sum_{i=1}^{n} y_{i} \chi_{\alpha_{i}}$, where $\chi_{\alpha_{i}}(t)=1$ if $t \in \alpha_{i}$ and $\chi_{\alpha_{i}}(t)=0$ if $t \notin \alpha_{i}$. A function $f: T \rightarrow Y$ is said to be $\mu$-measurable if there exists a sequence of simple functions $f_{n}: T \rightarrow Y$ such that $\lim _{n \rightarrow \infty}\left\|f_{n}(t)-f(t)\right\|=0$ for almost all $t \in T$. A $\mu$-measurable function $f: T \rightarrow Y$ is said to be Bochner integrable if there exists a sequence of simple functions $\left\{f_{n}\right\}(n=1,2, \ldots)$ such that

$$
\lim _{n \rightarrow \infty} \int_{T}\left\|f_{n}(t)-f(t)\right\| d \mu(t)=0
$$

In this case we define for each $E \in \tau$ the integral to be

$$
\int_{E} f(t) d \mu(t)=\lim _{n \rightarrow \infty} \int_{E} f_{n}(t) d \mu(t)
$$

It can be shown (see Diestel and Uhl [5, Theorem 2, p. 45]) that if $f: T \rightarrow Y$ is a $\mu$-measurable function then $f$ is Bochner integrable if and only if $\int_{T}\|f(t)\| d \mu(t)<$ $\infty$. We denote by $L_{1}(\mu, Y)$ the space of equivalence classes of $Y$-valued Bochner integrable functions $x: T \rightarrow Y$ normed by $\|x\|=\int_{T}\|x(t)\| d \mu(t)$. As was noted in 
Diestel and Uhl [5, p. 50], it can be easily shown that normed by the functional $\|\cdot\|$ above, $L_{1}(\mu, Y)$ becomes a Banach space.

A Banach space $Y$ has the Radon-Nikodym Property with respect to the measure space $(T, \tau, \mu)$ if for each $\mu$-continuous vector measure $G: \tau \rightarrow Y$ of bounded variation there exists $g \in L_{1}(\mu, Y)$ such that $G(E)=\int_{E} g(t) d \mu(t)$ for all $E \in \tau$. A Banach space $Y$ has the Radon-Nikodym Property (RNP) if $Y$ has the RNP with respect to every finite measure space. It is a standard result (see for instance [5, p. 98 or 6, p. 112]) that if $Y^{*}$ has the RNP then $\left(L_{1}(\mu, Y)\right)^{*}=L_{\infty}\left(\mu, Y^{*}\right)$.

Let $(T, \tau, \mu)$ be a finite measure space and $Y$ be a Banach space. The correspondence $\phi: T \rightarrow 2^{Y}$ is said to be integrably bounded if there exists a map $g \in L_{1}(\mu)$ such that for almost all $t$ in $T, \sup \{\|x\|: x \in \phi(t)\} \leq g(t)$. We denote by $\mathcal{L}_{\phi}$ the set of all $Y$-valued Bochner integrable selections of $\phi: T \rightarrow 2^{Y}$, i.e., $\mathcal{L}_{\phi}=\left\{x \in L_{1}(\mu, Y): x(t) \in \phi(t)\right.$ for almost all $t$ in $\left.T\right\}$. Following Aumann [2] the integral of the correspondence $\phi$ is defined as

$$
\int_{T} \phi(t) d \mu(t)=\left\{\int_{T} x(t) d \mu(t): x \in \mathcal{L}_{\phi}\right\} .
$$

It is a standard result (see for instance Himmelberg [11]) that if $T$ is a complete finite measure space, $Y$ is a separable Banach space, and $\phi: T \rightarrow 2^{Y}$ is a nonempty-valued correspondence with a measurable graph (or equivalently $\phi(\cdot)$ is lower measurable and closed valued), then $\phi(\cdot)$ admits a measurable selection, i.e., there exists a measurable function $f: T \rightarrow Y$ such that $f(t) \in \phi(t)$ for almost all $t$ in $T$. By virtue of this result and provided that $\phi$ is integrably bounded, we can conclude that $\mathcal{L}_{\phi} \neq \varnothing$ and therefore $\int_{T} \phi(t) d \mu(t) \neq \varnothing$.

Finally we wish to note that Diestel's Theorem [4] tells us that if $K$ is a nonempty, weakly compact, convex subset of a separable Banach space $Y$ (or more generally if $K: T \rightarrow 2^{Y}$ is an integrably bounded, nonempty, weakly compact convex valued correspondence, see [16, p. 570]) then $\mathcal{L}_{K}$ is weakly compact in $L_{1}(\mu, Y)$. Diestel's Theorem (which is an easy consequence of James's Theorem as Khan [14] showed) will play an important role in the sequel.

\section{The Main Theorem.}

3.1 An approximate version of the Fatou Lemma in infinite-dimensional spaces. Below we state the main result of the paper, i.e., an approximate version of the Fatou Lemma in infinite-dimensional spaces.

MAIN THEOREM. Let $(T, \tau, \mu)$ be a complete finite atomless measure space and $Y$ be a separable Banach space whose dual $Y^{*}$ has the RNP. Let $\phi_{n}: T \rightarrow 2^{Y}(n=$ $1,2, \ldots)$ be a sequence of nonempty closed valued correspondences such that:

(3.i) For all $n(n=1,2, \ldots), \phi_{n}(t) \subset X(t)$ for all $t \in T$, where $X: T \rightarrow 2^{Y}$ is an integrably bounded, weakly compact, convex, nonempty valued correspondence, and (3.ii) Ls $\phi_{n}(\cdot)(n=1,2, \ldots)$ is lower measurable.

Then

$$
\operatorname{Ls} \int_{T} \phi_{n}(t) d \mu(t) \subset \mathrm{cl} \int_{T} \operatorname{Ls} \phi_{n}(t) d \mu(t) .
$$

PROOF. Let $x \in \operatorname{Ls} \int_{T} \phi_{n}(t) d \mu(t)$; we will show that $x \in \operatorname{cl} \int_{T} \operatorname{Ls} \phi_{n}(t) d \mu(t)$. Now $x \in \operatorname{Ls} \int_{T} \phi_{n}(t) d \mu(t)$ implies that there exists $x_{k} \in \int_{T} \phi_{n_{k}}(t) d \mu(t)$ such that $x_{k}$ converges weakly to $x$. By the definition of the integral there exists 
$f_{k} \in \mathcal{L}_{\phi_{n_{k}}}$ such that $x_{k}=\int_{T} f_{k}(t) d \mu(t)$. Notice that $\mathcal{L}_{\phi_{n_{k}}} \subset \mathcal{L}_{X}$ and by assumption the correspondence $X: T \rightarrow 2^{Y}$ is integrably bounded, nonempty, weakly compact and convex valued. Hence, by Diestel's Theorem, $\mathcal{L}_{X}$ is weakly compact in $L_{1}(\mu, Y)$. Consequently, without loss of generality we may assume (otherwise pass to a subsequence) that $f_{k}$ converges weakly to $f \in \mathcal{L}_{X}$ (recall the Eberlein-Smulian theorem). Therefore, $x=\int_{T} f(t) d \mu(t)$. We now show that $f(t) \in \overline{\operatorname{con}} \operatorname{Ls}\left\{f_{k}(t)\right\}$ for almost all $t \in T$. Notice that $f_{k}$ converges weakly to $f$ implies that $\left\langle p, f_{k}\right\rangle$ converges to $\langle p, f\rangle$ for any $p \in\left(L_{1}(\mu, Y)\right)^{*}=L_{\infty}\left(\mu, Y^{*}\right)$. Since $\left\langle p, f_{k}\right\rangle=\int_{T}\left\langle p(t), f_{k}(t)\right\rangle d \mu(t)$ and $\langle p, f\rangle=\int_{T}\langle p(t), f(t)\rangle d \mu(t)$ we conclude that $\int_{T}\left\langle p(t), f_{k}(t)\right\rangle d \mu(t)$ converges to $\int_{T}\langle p(t), f(t)\rangle d \mu(t)$. Define the functions $h_{k}: T \rightarrow \mathbf{R}$ and $h: T \rightarrow \mathbf{R}$ by $h_{k}(t)=\left\langle p(t), f_{k}(t)\right\rangle$ and $h(t)=\langle p(t), f(t)\rangle$ respectively. Since $f_{k}$ lies in a weakly compact set, $h_{k}$ is bounded and uniformly integrable. Moreover, $h_{k}$ converges weakly to $h$. To see this let $g \in\left(L_{1}(\mu)\right)^{*}=L_{\infty}(\mu)$ and let $M=\|g\|_{\infty}$. Then

$$
\begin{aligned}
\left|\int_{T} g(t)\left(h_{k}(t)-h(t)\right) d \mu(t)\right| & \leq M\left|\int_{T}\left(h_{k}(t)-h(t)\right) d \mu(t)\right| \\
& =M\left|\int_{T}\left(\left\langle p(t), f_{k}(t)\right\rangle-\langle p(t), f(t)\rangle\right) d \mu(t)\right|,
\end{aligned}
$$

and (3.1) can become arbitrarily small since $\int_{T}\left\langle p(t), f_{k}(t)\right\rangle d \mu(t)$ converges to $\int_{T}\langle p(t), f(t)\rangle d \mu(t)$. By Proposition C in [1], for almost all $t \in T$,

$$
\begin{aligned}
h(t) & =\langle p(t), f(t)\rangle \in \operatorname{con} \operatorname{Ls}\left\{h_{k}(t)\right\}=\operatorname{con} \operatorname{Ls}\left\{\left\langle p(t), f_{k}(t)\right\rangle\right\} \\
& \subset \overline{\operatorname{con}} \operatorname{Ls}\left\{\left\langle p(t), f_{k}(t)\right\rangle\right\}=\left\langle p(t), \overline{\operatorname{con} L s}\left\{f_{k}(t)\right\}\right\rangle .
\end{aligned}
$$

Hence for almost all $t \in T,\langle p(t), f(t)\rangle \in\left\langle p(t), \overline{\operatorname{con}} \operatorname{Ls}\left\{f_{k}(t)\right\}\right\rangle$ and so

$$
f(t) \in \overline{\operatorname{con}} \operatorname{Ls}\left\{f_{k}(t)\right\} \quad \text { for almost all } t \in T \text {. }
$$

It follows from (3.2) that $f(t) \in \overline{\operatorname{con}} \mathrm{Ls} \phi_{n}(t)$ for almost all $t \in T$ and, consequently, $x=\int_{T} f(t) d \mu(t) \in \int_{T} \overline{\operatorname{con}} \operatorname{Ls} \phi_{n}(t) d \mu(t)$. Since, by assumption, Ls $\phi_{n}(\cdot)$ is lower measurable and $(T, \tau, \mu)$ is a complete finite atomless measure space, it follows from Theorem 1 in [13] that $^{1}$

$$
\int_{T} \overline{\operatorname{con}} \mathrm{Ls} \phi_{n}(t) d \mu(t)=\operatorname{cl} \int_{T} \operatorname{Ls} \phi_{n}(t) d \mu(t) .
$$

Therefore, $x \in \operatorname{cl~} \int_{T} \operatorname{Ls} \phi_{n}(t) d \mu(t)$ and this completes the proof of the Main Theorem.

REMARK 3.1. The assumption that $Y^{*}$ has the RNP was only used to ensure that $\left(L_{1}(\mu, Y)\right)^{*}=L_{\infty}\left(\mu, Y^{*}\right)$ and consequently for $f \in L_{1}(\mu, Y)$ and $p \in$ $L_{\infty}\left(\mu, Y^{*}\right)$ we can write $\langle p, f\rangle=\int_{T}\langle p(t), f(t)\rangle d \mu(t)$. However, the Main Theorem remains true if $Y$ is an arbitrary separable Banach space. In particular, in this case as Khan and Majumdar [15] noted, $p$ can be represented by a function $\psi: T \rightarrow Y^{*}$ such that $\langle\psi, y\rangle$ is measurable for every $y \in Y$ and $\|\psi\| \in L_{\infty}(\mu)$ (Dinculeanu [6, p.

\footnotetext{
${ }^{1}$ It should be noted that Theorem 1 of Khan [13] is true in separable Banach spaces and Banach spaces whose dual have the RNP.
} 
112]). Hence, $\langle p, f\rangle=\int_{T}\langle\psi(t), f(t)\rangle d \mu(t)$ and the argument of the Main Theorem used to prove that $h_{n}$ converges weakly to $h$ remains the same. ${ }^{2}$

3.2 An exact version of the Fatou Lemma in infinite-dimensional spaces. We now show how our Main Theorem can be used to obtain an exact version of the Fatou Lemma in infinite-dimensional spaces. We will first need the following lemma.

LEMMA 3.1. Let $(T, \tau, \mu)$ be a complete finite measure space and $Y$ be a separable Banach space. Let $\phi: T \rightarrow 2^{Y}$ be a nonempty, closed, convex valued correspondence such that $\phi(t) \subset X(t)$ for all $t \in T$, where $X: T \rightarrow 2^{Y}$ is an integrably bounded, nonempty, weakly compact, convex valued correspondence. Then

$$
\mathrm{cl} \int_{T} \phi(t) d \mu(t)=\int_{T} \phi(t) d \mu(t) .
$$

ProOF. Notice that $\mathcal{L}_{\phi} \subset \mathcal{L}_{X}$ and the latter set is weakly compact in $L_{1}(\mu, Y)$ (recall Diestel's Theorem). Since $\phi(\cdot)$ is norm closed and convex, so is $\mathcal{L}_{\phi}$. Hence, $\mathcal{L}_{\phi}$ is a weakly closed subset of $\mathcal{L}_{X}$ and consequently $\mathcal{L}_{\phi}$ is weakly compact. ${ }^{3}$ Define the mapping $\psi: L_{1}(\mu, Y) \rightarrow Y$ by $\psi(x)=\int_{T} x(t) d \mu(t)$. Certainly, $\psi$ is linear and norm continuous. By Theorem 15 in Dunford-Schwartz [8, p. 422], $\psi$ is also weakly continuous. Therefore, $\psi\left(\mathcal{L}_{\phi}\right)=\left\{\psi(x): x \in \mathcal{L}_{\phi}\right\}=\int_{T} \phi(t) d \mu(t)$ is weakly compact and we conclude that

$$
\operatorname{cl} \int_{T} \phi(t) d \mu(t)=\int_{T} \phi(t) d \mu(t)
$$

REMARK 3.2. The assumption that $\phi: T \rightarrow 2^{Y}$ is convex valued cannot be relaxed from the above lemma. In particular, Rustichini [17] has shown that without convex valueness of $\phi$, Lemma 3.1 is false.

Now combining the Main Theorem with the above lemma we can obtain an exact version of the Fatou Lemma in infinite-dimensional spaces.

THEOREM 3.1. Let $(T, \tau, \mu)$ be a complete finite atomless measure space and $Y$ be a separable Banach space. Let $\phi_{n}: T \rightarrow 2^{Y}(n=1,2, \ldots)$ be a sequence of nonempty closed valued correspondences such that assumption (3.i) of the Main Theorem is satisfied. Moreover, suppose that $\mathrm{Ls} \phi_{n}(\cdot)$ is closed and convex valued. Then

$$
\operatorname{Ls} \int_{T} \phi_{n}(t) d \mu(t) \subset \int_{T} \operatorname{Ls} \phi_{n}(t) d \mu(t) .
$$

PROOF. It follows from the Main Theorem and Remark 3.1 that

$$
\operatorname{Ls} \int_{T} \phi_{n}(t) d \mu(t) \subset \operatorname{cl} \int_{T} \operatorname{Ls} \phi_{n}(t) d \mu(t) .
$$

\footnotetext{
${ }^{2}$ The Main Theorem also remains true if $Y$ is a Banach space whose dual $Y^{*}$ has the RNP, provided that (3.i) is replaced by

(3.i)' For all $n, \phi_{n}(t) \subset X$ for all $t \in T$ where $X$ is a weakly compact, convex, nonempty subset of $Y$.

In this case $\mathcal{L}_{X}$ is still weakly compact (see Diestel [4] or Khan [14]) and the proof of the Main Theorem remains unchanged.

${ }^{3}$ Recall that it is a consequence of the separation theorem that the norm and weak topologies coincide on closed convex sets.
} 
Since $\operatorname{Ls} \phi_{n}(\cdot)$ is closed and convex by virtue of Lemma 3.1 we have that

$$
\operatorname{Ls} \int \phi_{n}(t) d \mu(t) \subset \operatorname{cl} \int_{T} \operatorname{Ls} \phi_{n}(t) d \mu(t)=\int_{T} \operatorname{Ls} \phi_{n}(t) d \mu(t) .
$$

This completes the proof of the theorem. ${ }^{4}$

REMARK 3.3. Notice that the counterexample of Rustichini [17] on the failure of the Fatou Lemma in infinite-dimensional spaces does not upset Theorem 3.1 since it does not satisfy the assumption that $\operatorname{Ls} \phi_{n}(\cdot)$ is convex valued.

4. Integration preserves upper-semicontinuity. If $X, Y$ are topological spaces we will say that $\psi: X \rightarrow 2^{Y}$ has a closed graph if $G_{\psi}$ is closed in $X \times Y$. In our setting below, $X$ is a metric space and $Y$ is a Banach space. In this latter setting $\psi: X \rightarrow 2^{Y}$ is said to have a weakly closed graph if $G_{\psi}$ is closed in the product topology $X \times Y$, where $Y$ is endowed with the weak topology. Following Aumann [3] we will say that the correspondence $\psi: X \rightarrow 2^{Y}$ is weakly u.s.c. if $\psi$ has a weakly closed graph.

Let $T$ be a measure space, $P$ be a metric space, $Y$ be a Banach space, and $\phi: T \times P \rightarrow 2^{Y}$ be a nonempty valued correspondence. For $Y=\mathrm{R}^{l}$ Aumann [2] showed that if $\phi(t, \cdot)$ is u.s.c. for each fixed $t \in T$, then so is $\int_{T} \phi(t, \cdot) d \mu(t)$. Aumann [2] proved this result by means of the Fatou Lemma in several dimensions (a similar result can also be found in Hildenbrand $[9$, p. 73]). An alternative elementary proof of the same result was also given by Aumann [3]. Below we show that an infinitedimensional version of the above fact follows directly from our Main Theorem.

THEOREM 4.1. Let $(T, \tau, \mu)$ be a complete, finite, atomless measure space, $P$ be a metric space, and $Y$ be a separable Banach space. Let $\phi: T \times P \rightarrow 2^{Y}$ be a nonempty convex valued correspondence such that:

(4.i) For each fixed $t \in T, \phi(t, \cdot)$ is weakly u.s.c.,

(4.ii) for all $(t, p) \in T \times P, \phi(t, p) \subset X(t)$, where $X: T \rightarrow 2^{Y}$ is an integrably bounded, weakly compact, convex, nonempty valued correspondence, and

(4.iii) for each fixed $p \in P$, Ls $\phi(\cdot, p)$ is lower measurable.

Then $\int_{T} \phi(t, \cdot) d \mu(t)$ is weakly u.s.c.

PROOF. Let $p_{n}(n=1,2, \ldots)$ be a sequence in $P$ converging to $p$. We must show that $\operatorname{Ls} \int \phi\left(\cdot, p_{n}\right) \subset \int \phi(\cdot, p)$. Since, by assumption, $\phi(t, \cdot)$ is weakly u.s.c. for each fixed $t \in T$, we have that for all $t \in T$, Ls $\phi\left(t, p_{n}\right) \subset \phi(t, p)$. Therefore,

$$
\int_{T} \operatorname{Ls} \phi\left(t, p_{n}\right) d \mu(t) \subset \int_{T} \phi(t, p) d \mu(t) .
$$

By virtue of Lemma 3.1, (4.1) can be written as

$$
\operatorname{cl} \int_{T} \operatorname{Ls} \phi\left(t, p_{n}\right) d \mu(t) \subset \int_{T} \phi(t, p) d \mu(t) .
$$

It follows now from the Main Theorem that

$$
\operatorname{Ls} \int_{T} \phi\left(t, p_{n}\right) d \mu(t) \subset \operatorname{cl} \int_{T} \operatorname{Ls} \phi\left(t, p_{n}\right) d \mu(t) \subset \int_{T} \phi(t, p) d \mu(t) .
$$

This completes the proof of the theorem.

\footnotetext{
${ }^{4}$ It should be noted that $\int_{T} \operatorname{Ls} \phi_{n}(t) d \mu(t)=\int_{T} \overline{\operatorname{con}} \operatorname{Ls} \phi_{n}(t) d \mu(t)$ since Ls $\phi_{n}(\cdot)$ is convex valued, and in this case Khan's approximate version of the Lyapunov-Richter Theorem is not needed in the proof of the Main Theorem. Therefore, Theorem 4.1 remains true without the nonatomicity assumption on the measure space.
} 
REMARK 4.1. It should be noted that in the case that instead of correspondences we deal with functions, i.e., $\phi: T \times P \rightarrow Y$ is a function such that $\phi$ is integrably bounded and for each fixed $t \in T, \phi(t, \cdot)$ is continuous, then we can automatically conclude (by virtue of the Lebesgue Dominated Convergence Theorem $[5, \mathrm{p} .45])$, that $\int_{T} \phi(t, \cdot) d \mu(t)$ is also continuous.

REMARK 4.2. Results similar to Theorem 4.1 have been obtained in Khan [12] and Yannelis [20] (among others). In particular, in the above works it has been shown that if a correspondence is u.s.c., then the set of its integrable selections is also u.s.c.

5. Relationship with the Khan-Majumdar work. Our Main Theorem, as well as the idea of the proof, were both inspired by reading the work of Khan [13, 14] and Khan and Majumdar [15]. The contribution of our paper is to simplify and slightly generalize the theorem of Khan and Majumdar by integrating three major results: Diestel's Theorem on weak compactness, Artstein's Lemma, and Khan's approximate version of the Lyapunov-Richter Theorem. In particular, the following corollary of our Main Theorem is the infinite-dimensional version of the Fatou Lemma proved by Khan and Majumdar.

COROLlaRY 5.1. Let $(T, \tau, \mu)$ be a finite, complete, atomless measure space and $Y$ be a separable Banach space. Let $f_{n}(n=1,2, \ldots)$ be a sequence of functions from $L_{1}(\mu, Y)$ such that for all $n, f_{n}(t) \in K$ for all $t$ in $T$, where $K$ is a nonempty, weakly compact, convex subset of $Y$ and suppose that the weak limit $\mathrm{w}-$ $\operatorname{Lim}_{n \rightarrow \infty} \int_{T} f_{n}(t) d \mu(t)$ exists. Then for all $\varepsilon>0$ there exists $f \in L_{1}(\mu, Y)$ such that:

(5.i) $f(t) \in \operatorname{Ls}\left\{f_{n}(t)\right\}$ for almost all $t$ in $T$, and

(5.ii) $\left\|\int_{T} f(t) d \mu(t)-\mathrm{w}-\operatorname{Lim}_{n \rightarrow \infty} \int_{T} f_{n}(t) d \mu(t)\right\|<\varepsilon$.

ProOF. The proof follows directly from the Main Theorem by setting $\phi_{n}(t)=$ $\left\{f_{n}(t)\right\}(n=1,2, \ldots)$ for all $t$ in $T$ and $X(t)=K$ for all $t$ in $T$. One only needs to use Lemma 4.1 in Dsosu and Shaozhong [7] in order to show that $\operatorname{Ls}\left\{f_{n}\right\}$ is lower measurable and thus to conclude that assumption (3.ii) of the Main Theorem is satisfied.

Notice that Corollary 5.1 remains true if $Y$ is a Banach space whose dual $Y^{*}$ has the RNP (recall footnote 2).

NOTE ADDED IN PROOF. I wish to thank David Schmeidler for informing me that Erik Balder using different arguments, has independently proved a version of our Main Theorem. Professor Balder's work in forthcoming in the J. Math. Anal. Appl. The author has benefited from the comments and suggestions of M. Ali Khan and Aldo Rustichini.

\section{REFERENCES}

1. Z. Artstein, A note on Fatou's Lemma in several dimensions, J. Math. Econom. 6 (1979), 277-282.

2. R. J. Aumann, Integrals of set-valued functions, J. Math. Anal. Appl. 12 (1965), 1-12.

3. _ An elementary proof that integration preserves upper-semicontinuity, J. Math. Econom. 3 (1976), 15-18.

4. J. Diestel, Remarks on weak compactness in $L_{1}(\mu, X)$, Glasgow Math. J. 18 (1977), 87-91.

5. J. Diestel and J. Uhl, Vector measures Math. Surveys, no. 15, Amer. Math. Soc., Providence, R.I., 1977. 
6. N. Dinculeanu, Linear opertions on $L_{p}$-spaces, Vector and Operator Valued Measures and Applications (D. Tucker and H. Maynard, eds.), Academic Press, New York, 1973.

7. L. Dsosu and C. Shaozhong, On fixed point theorems for random set-valued maps, Nonlinear Anal. 7 (1983), 309-323.

8. N. Dunford and J. T. Schwartz, Linear operators, Part I, Interscience, New York, 1958.

9. W. Hildenbrand, Core and equilibria of a large economy, Princeton Univ. Press, Princeton, N.J., 1974.

10. W. Hildenbrand and J. Mertens, On Fatou's Lemma in several dimensions, Z. Wahrsch. Verw. Gebiete 17, Springer, 1971, pp. 151-155.

11. C. J. Himmelberg, Measurable relations, Fund. Math. 87 (1975), 53-72.

12. M. Ali Khan, Equilibrium points of nonatomic games over a Banach space, Trans. Amer. Math. Soc. 29 (1986), 737-749.

13. _ On the integration of set-valued mappings in a nonreflexive Banach space. II, Simon Stevin 59 (1985), 257-267.

14. __ An alternative proof of Diestel's Theorem, Glasgow Math. J. 25 (1984), 45-46.

15. M. Ali Khan and M. Majumdar, Weak sequential convergence in $L_{1}(\mu, X)$ and an approximate version of Fatou's Lemma, J. Math. Anal. Appl. 114 (1986), 569-573.

16. N. S. Papageorgiou, Representation of set valued operators, Trans. Amer. Math. Soc. 292 (1985), 557-572.

17. A. Rustichini, A counterexample to Fatou's Lemma in infinite dimensional spaces, Department of Math., Univ. of Minnesota, 1986.

18. D. Schmeidler, Fatou's Lemma in several dimensions, Proc. Amer. Math. Soc. 24 (1970), 300-306.

19. J. Uhl, The range of a vector-valued measure, Proc. Amer. Math. Soc. 23 (1969), 158-163.

20. N. C. Yannelis, Equilibria in noncooperative models of competition, J. Econom. Theory 41 (1987), 96-111.

DePARTMENT OF ECONOMICS, UNIVERSity of Minnesota, Minneapolis, MinNESOTA 55455

Department of Economics, TUlane UNiVERSity, NeW ORLEANS, LOUisiana 70118 (Current address) 